\title{
Real-World Outcomes and Costs Following 6 Months of Treatment with the Long-Acting Injectable (LAI) Aripiprazole Lauroxil for the Treatment of Schizophrenia
}

\author{
John Lauriello ${ }^{1}$ Peter J. Weiden ${ }^{2} \cdot$ Carole D. Gleeson $^{2} \cdot$ Ankit Shah $^{2} \cdot$ Luke Boulanger $^{3} \cdot$ Krutika Jariwala-Parikh $^{3}$. \\ Elizabeth Hedgeman ${ }^{3}$. Amy K. O'Sullivan ${ }^{2}$
}

Accepted: 17 July 2021 / Published online: 21 September 2021

(c) The Author(s) 2021

\begin{abstract}
Background Continuous antipsychotic therapy is recommended as part of long-term maintenance treatment of schizophrenia, and gaps in antipsychotic treatment have been associated with increased risks of relapse and rehospitalization. Because the use of long-acting injectable (LAI) antipsychotics may reduce the likelihood of undetected medication gaps, initiating an LAI medication may affect resource utilization and costs. The LAI aripiprazole lauroxil (AL) was approved in the United States (US) in 2015 for the treatment of schizophrenia in adults.

Objective The objective of this retrospective observational cohort study was to examine treatment patterns, resource utilization, and costs following initiation of AL for the treatment of schizophrenia in adults.

Methods A retrospective analysis of Medicaid claims data identified a cohort of patients $(N=485)$ starting AL shortly after Food and Drug Administration approval in October 2015. Treatment patterns, resource utilization, and costs were compared 6 months before and after treatment initiation. Subgroup analyses were conducted based on the type of antipsychotic (LAI, oral, or none) received before initiation of AL.

Results Over 6 months of follow-up, patients received an average of 4.6 injections out of a maximum of six (77\%). After initiating AL, all-cause inpatient admissions decreased by $22.4 \%$; other significant reductions were observed in mental health-related admissions and emergency room (ER) visits. All-cause inpatient costs decreased by an average of US $\$ 2836$ per patient $(p<0.05)$ in the 6-month post-AL period, whereas outpatient pharmacy costs increased by US $\$ 4121(p<0.05)$, resulting in no significant difference in overall costs between the pre- and post-AL periods. The subgroup of patients who had been prescribed an oral antipsychotic before starting AL had significant reductions in proportion of patients with inpatient and ER visits and costs, but also reported a significant increase in pharmacy costs.

Conclusions AL was associated with a significant reduction in inpatient costs and an increase in outpatient pharmacy costs, resulting in no changes in total healthcare costs over 6 months. The adherence rate and reductions in inpatient use may indicate the potential for greater clinical stability among patients initiated on AL compared with their previous treatment.
\end{abstract}

Peter J. Weiden, Carole D. Gleeson and Ankit Shah: Employed by Alkermes, Inc., at the time this research was conducted.

John Lauriello

John.lauriello@jefferson.edu

1 Department of Psychiatry and Human Behavior, Jefferson Health-Sidney Kimmel Medical College, Thomas Jefferson University, 33 S 9th St, Ste 210, Philadelphia, PA, USA

2 Alkermes, Waltham, MA, USA

3 IBM Watson Health, Cambridge, MA, USA

\section{Introduction}

A leading cause of disability worldwide, schizophrenia was identified in 2017 as one of the top 20 contributors to years lived with disability among both females and males [1]. At that time, the prevalence of schizophrenia was estimated at approximately 20 million and was projected to increase in a growing and aging global population [2,3]. Annual total costs of schizophrenia are also high, ranging from $0.02 \%$ (the United Kingdom [UK]) to $1.65 \%$ (Sweden) of the gross domestic product (United States [US] dollars [US\$] 2013) in 15 countries studied [4]. In the US, schizophrenia is among the top 30 causes of disability [5]. It is associated with a 


\section{Key Points}

Aripiprazole lauroxil (AL) is an atypical long-acting injectable antipsychotic that was approved in the United States for the treatment of schizophrenia in adults in October 2015.

We examined treatment patterns, healthcare resource utilization and costs before and after initiation of AL for the treatment of schizophrenia in a cohort started on AL shortly after US Food and Drug Administration approval.

Results of the study suggest that patients have good persistence with AL and that inpatient costs may be reduced while outpatient pharmacy costs increase.

The greatest reduction in subsequent inpatient and emergency room use was observed in the subgroup that had been prescribed an oral antipsychotic before starting AL.

large disease burden including shortened life expectancy, high rates of lifetime disability, and growing economic costs [6]. The total excess annual direct medical cost per patient with schizophrenia insured by Medicaid has been estimated at US\$23,455 (US\$2013) [6].

Continuous antipsychotic therapy is widely recommended to be part of long-term maintenance treatment of schizophrenia, where delaying or preventing relapse is an essential treatment goal. Conversely, discontinuation and disruptions in antipsychotic therapy are major risk factors for symptom exacerbation and relapse [7-10]. Unfortunately, both intentional and inadvertent medication discontinuation are frequent events over the course of long-term treatment. Further, these medication interruptions during oral therapy often go undetected in clinical practice $[11,12]$. Given the potentially dire consequences of relapse, encouraging patients to remain on antipsychotic therapy represents an important long-term goal in schizophrenia management $[10,13,14]$. This is a significant challenge, given that as many as $75 \%$ of patients discontinue their antipsychotic at some point within 2 years of hospital discharge [15-17]. While there is no single solution to this problem, long-acting injectable (LAI) antipsychotics, relative to their oral counterparts, may reduce the likelihood of unintentional medication gaps. LAI medications also provide reliable information to the physician as to the actual adherence status from one visit to the next. That is, failure to appear or accept the LAI antipsychotic alerts the clinician that nonadherence is occurring as an issue in real time [18-20]. While improved adherence is a goal of treatment with LAI antipsychotics, it is also important to understand the impact on resource utilization and costs.

Aripiprazole lauroxil (AL) is an atypical LAI antipsychotic that was approved in the US for the treatment of schizophrenia in adults in October 2015 [21]. The objective of this study was to examine early experience (i.e., less than 2 years after approval) with AL in schizophrenia, including treatment patterns of AL use and healthcare resource utilization and costs in the 6 months before and after initiation of AL therapy.

\section{Methods}

\subsection{Data Source}

This retrospective, observational cohort study utilized administrative claims data from the MarketScan ${ }^{\circledR}$ MultiState Medicaid Database spanning the period between April 1, 2015, and December 31, 2017. This database contains the pooled healthcare experience of approximately 47.3 million Medicaid enrollees, living in multiple geographically dispersed states between 1999 and 2017, including 13.5 million lives in 2017. Each patient record contains longitudinal data including inpatient services, outpatient services, and prescription drug claims, as well as information on longterm care and other medical care. All database records are statistically de-identified and certified to be fully compliant with US patient confidentiality requirements set forth in the Health Insurance Portability and Accountability Act (HIPAA) of 1996. Because this study used only de-identified patient records and did not involve the collection, use, or transmittal of individually identifiable data, it was exempted from institutional review board approval. All study data were obtained using International Classification of Diseases, 9th and 10th Revision, Clinical Modification (ICD-9-CM and ICD-10-CM) codes, Healthcare Common Procedure Coding System (HCPCS) codes, and National Drug Codes (NDCs).

\subsection{Patient Selection}

Patients included in the study met the following inclusion criteria:

- Aged 18 years and over

- At least one claim for one of the AL dose strengths available between October 1, 2015, and June 30, 2017 (i.e., $441 \mathrm{mg}, 662 \mathrm{mg}$, or $882 \mathrm{mg}$ )

- At least one claim with a diagnosis code for schizophrenia (ICD-9-CM: 295.xx, 298.9; ICD-10-CM: F20.x, F25.x, or F29) on or during the 12 months before the first claim for AL

- Continuous enrollment with medical, pharmacy, and mental health and substance abuse coverage for at least 6 months before (baseline period) and 6 months after (follow-up period) the date of the first pharmacy or medical claim for AL (hereafter termed the "index date") 
Patients with dual Medicaid/Medicare enrollment coverage during their baseline or follow-up period were excluded to ensure that all healthcare claims could be tracked through Medicaid data.

\subsection{Demographic and Clinical Characteristics}

Baseline demographic characteristics were assessed on the index date. Clinical characteristics were assessed during the 6-month baseline period, including mental health conditions and physical comorbidities using the Deyo-Charlson Comorbidity Index (DCI) [22] and non-antipsychotic psychiatric medication use. The DCI, an administrative claims-based modification of the Charlson Comorbidity Index, is an aggregate measure of comorbidity expressed as a numeric score that serves as a proxy for overall patient health status; larger scores are associated with a higher comorbidity burden [22].

\subsection{Treatment Patterns}

Treatment patterns for AL were measured during the 6-month follow-up period and included total number of AL injections received over the 6-month follow-up period, duration of AL exposure, proportion of days covered (PDC), proportion of patients who discontinued, and time to discontinuation. For the 6-month follow-up period, we assumed a monthly prescribing cadence and therefore used six injections as representative of the maximum number of injections that could have been received.

At the time the data for this study were collected, there were four approved dosage regimen options for AL: 441 $\mathrm{mg}, 662 \mathrm{mg}$, or $882 \mathrm{mg}$ administered monthly or an 882$\mathrm{mg}$ dose every 6 weeks. Initiation of AL at the time the study was conducted required 21 consecutive days of oral aripiprazole to be given in conjunction with the first $\mathrm{AL}$ injection. Once AL is started, missed dose guidance per the labeling for Aristada injection intervals notes if and when patients are late for their next AL injection, the 441-mg injection can extend up to 6 weeks after the last injection (e.g., an additional 2 weeks on a monthly schedule) and the 662-mg and 882-mg injections can extend up to a total of 8 weeks (e.g., an additional 4 weeks for patients on a monthly schedule and 2 weeks for patients on an every 6 -week schedule). These intervals were used in determining time until discontinuation.

The duration of drug exposure was calculated as the sum of the days with medication on board, including the maximum length of time permitted before re-starting with oral supplementation is required (i.e., 45 days for $441 \mathrm{mg}$ administered monthly, 60 days for $662 \mathrm{mg}$ administered monthly, and $882 \mathrm{mg}$ administered monthly or every 6 weeks). PDC was defined as the duration of drug exposure divided by the length of the follow-up (180 days). Discontinuation was defined as the absence of a subsequent AL claim after the maximum permissible interval for the previous AL dose. Time to discontinuation was defined as the number of days from the index date to the end of the last treatment interval; patients who did not discontinue treatment over follow-up were censored at 6 months (180 days).

\subsection{Healthcare Resource Utilization and Costs}

All-cause and mental health-related healthcare resource utilization, including inpatient services (inpatient admissions and length of stay), outpatient services (emergency room [ER] visits, physician office visits, laboratory tests, radiology tests, and miscellaneous healthcare services performed in an outpatient setting), and outpatient pharmacy claims, were measured over the 6-month baseline and follow-up periods. Utilization or costs were classified as mental health related if they corresponded to (1) medical claims with a diagnosis code for mental health and related conditions (primary position); (2) medical claims with HCPCS codes for antipsychotics, antidepressants, anxiolytics/hypnotics/sedatives, central nervous system (CNS) stimulants, anticholinergic agents, or antimanic drugs; or (3) outpatient pharmacy claims (NDCs) for antipsychotics, antidepressants, anxiolytics/hypnotics/sedatives, CNS stimulants, anticholinergic agents, or antimanic drugs. Medical services were defined as mental health related based on HCPCS codes for drug therapies. Associated costs were calculated in US dollars. All dollar estimates were inflated to 2017 dollars using the Medical Care Component of the Consumer Price Index [23].

\subsection{Subgroup Analyses}

Subgroup analyses were conducted according to type of antipsychotic medication patients were on during the 60 days prior to the first AL injection. Specifically, patients were assigned subgroups as follows: (1) at least one LAI antipsychotic claim (with or without an oral antipsychotic claim) in the 60 days preceding the index AL date ("recent LAI antipsychotic"); (2) at least one oral antipsychotic claim without any LAI antipsychotic claim in the 60 days preceding index ("recent oral antipsychotic"); and (3) claims for neither an LAI nor oral antipsychotic in the 60 days preceding index ("no recent antipsychotic"). LAI antipsychotic claims were based on injection given, whereas oral claims represent prescription fills. All outcome measures were assessed for the total cohort as well as by subgroup.

\subsection{Statistical Analysis}

Categorical variables were presented as the count and percentage in each category, and continuous variables were 
summarized by mean and standard deviation (SD). Differences between baseline and follow-up utilization and cost measures were analyzed using two-sided paired $t$ tests or Mann-Whitney tests, as appropriate. Because the study used a within-patient (pre-versus-post) design, each patient served as their own control with no adjustment for covariates. Differences in baseline characteristics between subgroups defined by prior antipsychotic use were tested using chi-squared tests for categorical variables and Student's $t$ test or analysis of variance for continuous variables. An alpha level of 0.05 was used to determine statistical significance for all tests.

\section{Results}

\subsection{Baseline Characteristics}

A total of 485 patients met the inclusion criteria for the analysis (Fig. 1). Among all patients, the mean (SD) age was 35.3 (12.1) years, and $58.1 \%$ were male (Table 1). Both mental and physical comorbidities were common.

In the 60 days prior to initiating AL, 217 patients (44.7\%) had filled a prescription for an LAI antipsychotic other than AL, 195 patients (40.2\%) had filled a prescription for an oral antipsychotic only, and 73 patients (15.1\%) had not filled any antipsychotic prescription (Table 1). During baseline, the proportions of patients with prescriptions for antidepressants or for anxiolytics, hypnotics, or sedatives differed significantly between subgroups $(p=0.011$ and $p<0.001$, respectively). For both categories, proportions were lowest among patients with no recent antipsychotic use. No other significant differences in baseline characteristics were observed between subgroups (Table 1).

\subsection{Treatment Patterns After AL Initiation}

The most common initial dose of AL was $882 \mathrm{mg}$ (48.0\%) followed by $662 \mathrm{mg}$ (35.9\%) and $441 \mathrm{mg}$ (16.1\%) (Table 2).

Among all patients, the mean number of doses (injections) received during the 6-month follow-up period was $4.6(77 \%$ of a maximum six injections possible during the follow-up period, assuming a monthly cadence as per the prescribing information). Over $40 \%$ (43.5\%) received six or more AL injections. Patients had an average of 136.0 days of drug exposure out of a possible 180 days, which resulted in a mean (SD) PDC of $0.76(0.31)$ (Table 2). By the end of the follow-up period, $34 \%$ of all patients had either discontinued or missed an AL injection visit. The mean (SD) time to discontinuation among all patients was 133.4 (59.6) days (Table 2).

Treatment patterns varied by prior antipsychotic exposure. Significant differences between groups were observed

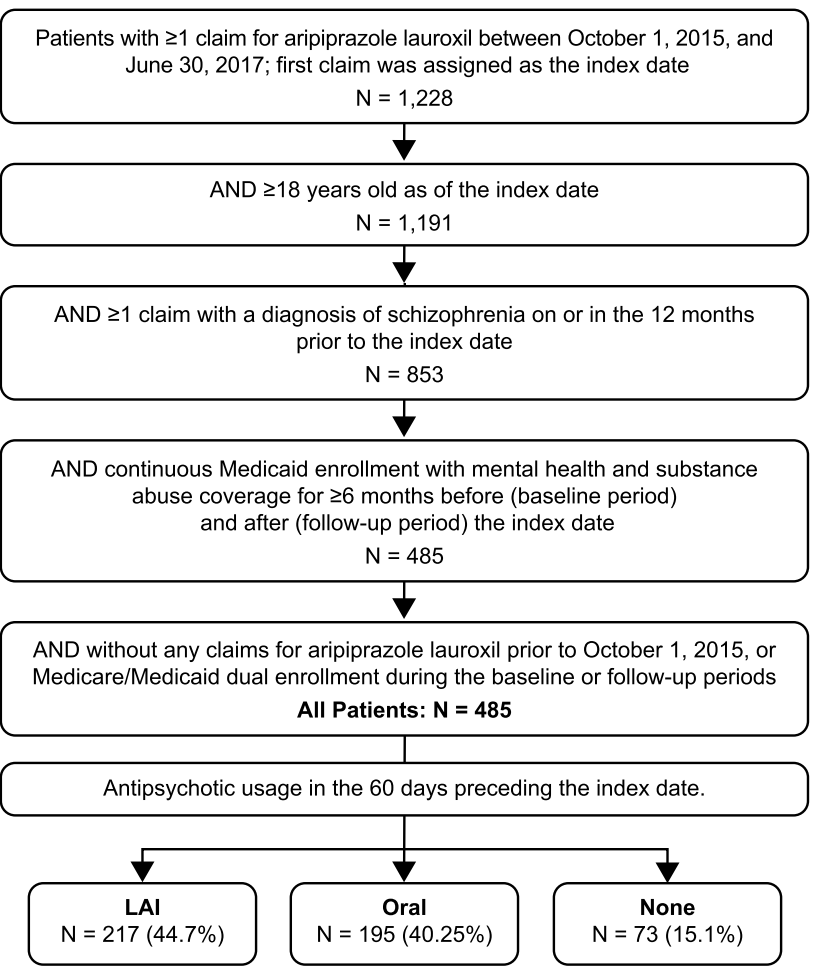

Fig. 1 Patient selection. $L A I$ long-acting injectable antipsychotic

in number of doses during follow-up $(p=0.001)$, proportion of patients receiving at least six doses of $\mathrm{AL}(p<0.001)$, $\operatorname{PDC}(p=0.039)$, and duration of AL exposure $(p=0.039)$. Patients who had been prescribed an LAI antipsychotic in the 60 days before starting AL went on to receive a larger mean number of $\mathrm{AL}$ doses, with a greater proportion receiving six or more, compared with patients in the recent oral antipsychotic or no recent antipsychotic subgroups (Table 2). Patients with recent LAI antipsychotic exposure also had a relatively longer mean duration of exposure and higher PDC with AL than patients in other subgroups (Table 2). Proportions of patients with prescriptions for antidepressants and for anxiolytics, hypnotics, or sedatives differed significantly between subgroups in the follow-up period ( $p$ $=0.004$ and $p<0.001$, respectively). For both medication categories, proportions were lowest among patients with no recent antipsychotic use.

\subsection{Healthcare Resource Utilization}

Table 3 summarizes patterns of all-cause and mental health-related healthcare resource utilization in the 6-month period before versus after initiation with $\mathrm{AL}$, for the total cohort and by subgroup. Supplemental Figure S1 displays the net change in utilization from baseline to follow-up, overall and by subgroup (see the electronic supplementary material). Below, we summarize trends for all-cause 
Table 1 Patient characteristics at baseline

\begin{tabular}{|c|c|c|c|c|c|}
\hline & \multicolumn{4}{|c|}{ Recent antipsychotic use ${ }^{a}$} & \multirow{2}{*}{$\begin{array}{l}\text { All patients } \\
N=485\end{array}$} \\
\hline & $\begin{array}{l}\text { Recent LAI } \\
N=217\end{array}$ & $\begin{array}{l}\text { Recent oral } \\
N=195\end{array}$ & $\begin{array}{l}\text { None } \\
N=73\end{array}$ & $P$ value & \\
\hline Age, mean (SD) & $35.4(11.6)$ & $34.7(12.5)$ & $36.3(12.6)$ & 0.594 & $35.3(12.1)$ \\
\hline Male, $n(\%)$ & $134(61.8)$ & $105(53.8)$ & $43(58.9)$ & 0.265 & $282(58.1)$ \\
\hline Race, $n(\%)$ & & & & 0.732 & \\
\hline White & $98(45.2)$ & $88(45.1)$ & $30(41.1)$ & & $216(44.5)$ \\
\hline Black & $94(43.3)$ & $79(40.5)$ & $33(45.2)$ & & $206(42.5)$ \\
\hline Hispanic & $5(2.3)$ & $4(2.1)$ & $0(0.0)$ & & $9(1.9)$ \\
\hline Other/unknown & $20(9.2)$ & $24(12.3)$ & $10(13.7)$ & & $54(11.1)$ \\
\hline DCI, mean (SD) & $0.6(1.0)$ & $0.6(1.0)$ & $0.5(1.3)$ & 0.759 & $0.6(1.1)$ \\
\hline \multicolumn{6}{|c|}{ Mental health-related comorbidities, $n(\%)$} \\
\hline Major depressive disorder & $68(31.3)$ & $76(39.0)$ & $19(26.0)$ & 0.086 & $163(33.6)$ \\
\hline Anxiety or panic disorder & $59(27.2)$ & $65(33.3)$ & $17(23.3)$ & 0.194 & $141(29.1)$ \\
\hline Drug dependence & $34(15.7)$ & $29(14.9)$ & $11(15.1)$ & 0.974 & $74(15.3)$ \\
\hline Alcohol dependence & $13(6.0)$ & $16(8.2)$ & $2(2.7)$ & 0.252 & $31(6.4)$ \\
\hline \multicolumn{6}{|l|}{ Psychiatric medication use, $n(\%)$} \\
\hline Antidepressants & $138(63.6)$ & $141(72.3)$ & $39(53.4)$ & 0.011 & $318(65.6)$ \\
\hline Anxiolytics/hypnotics/sedatives & $112(51.6)$ & $120(61.5)$ & $18(24.7)$ & $<0.001$ & $250(51.5)$ \\
\hline Anticholinergic agents & $84(38.7)$ & $66(33.8)$ & $19(26.0)$ & 0.134 & $169(34.8)$ \\
\hline Mood stabilizers & $72(33.2)$ & $73(37.4)$ & $21(28.8)$ & 0.375 & $166(34.2)$ \\
\hline CNS stimulants & $22(10.1)$ & $16(8.2)$ & $3(4.1)$ & 0.274 & $41(8.5)$ \\
\hline
\end{tabular}

Statistically significant values are in bold

$A L$ aripiprazole lauroxil, $C N S$ central nervous system, $D C I$ Deyo-Charlson Comorbidity Index, $L A I$ long-acting injectable, $S D$ standard deviation ${ }^{a}$ Recent antipsychotic use was in the 60-day period prior to initiation of AL

Table 2 Treatment patterns after initiation of aripiprazole lauroxil (AL)

\begin{tabular}{|c|c|c|c|c|c|}
\hline & \multicolumn{4}{|c|}{ Recent antipsychotic use } & \multirow{2}{*}{$\begin{array}{l}\text { All patients } \\
N=485\end{array}$} \\
\hline & $\begin{array}{l}\text { Recent LAI } \\
N=217\end{array}$ & $\begin{array}{l}\text { Recent oral } \\
N=195\end{array}$ & $\begin{array}{l}\text { None } \\
N=73\end{array}$ & $P$ value & \\
\hline Initial AL dosage strength given ${ }^{\mathrm{a}}, n(\%)$ & & & & $<0.001$ & \\
\hline $441 \mathrm{mg}$ & $19(8.8)$ & $51(26.2)$ & $8(11.0)$ & & $78(16.1)$ \\
\hline $662 \mathrm{mg}$ & $93(42.9)$ & $57(29.2)$ & $24(32.9)$ & & $174(35.9)$ \\
\hline $882 \mathrm{mg}^{\mathrm{b}}$ & $105(48.4)$ & $87(44.6)$ & $41(56.2)$ & & $233(48.0)$ \\
\hline PDC, mean (SD) & $0.79(0.29)$ & $0.72(0.32)$ & $0.74(0.33)$ & $\mathbf{0 . 0 3 9}$ & $0.76(0.31)$ \\
\hline Number of doses during follow-up, mean (SD) & $5.0(2.1)$ & $4.3(2.1)$ & $4.3(2.2)$ & 0.001 & $4.6(2.1)$ \\
\hline Proportion of patients receiving $\geq 6$ doses of $\mathrm{AL}$ & $116(53.5)$ & $72(36.9)$ & $23(31.5)$ & $<0.001$ & $211(43.5)$ \\
\hline Duration of AL exposure in days, mean (SD) & $143.0(53.0)$ & $129.0(58.4)$ & $133.6(60.2)$ & $\mathbf{0 . 0 3 9}$ & $136.0(56.6)$ \\
\hline Proportion of patients who discontinued, $n(\%)$ & $63(29.0)$ & $78(40.0)$ & $24(32.9)$ & 0.062 & $165(34.0)$ \\
\hline Time to first discontinuation in days, mean $(\mathrm{SD})^{\mathrm{c}}$ & $58.1(30.0)$ & $57.9(33.2)$ & $44.9(25.9)$ & 0.164 & $56.1(31.2)$ \\
\hline
\end{tabular}

Statistically significant values are in bold

$L A I$ long-acting injectable, $P D C$ proportion of days covered, $S D$ standard deviation

${ }^{a}$ No patients initiated at a dose of $1064 \mathrm{mg}$ as it was not yet approved during the years in which the analysis was conducted

${ }^{\mathrm{b}}$ The 882-mg dose was assumed to be the 4-week dose for all patients

${ }^{\mathrm{c}}$ Among patients who discontinued $\mathrm{AL}(n=134)$ 
inpatient, outpatient, and prescription drug utilization. Mental health-related healthcare utilization is described only if markedly different from all-cause utilization.

\subsubsection{Inpatient Utilization}

Among all patients, the mean number of all-cause inpatient admissions per patient decreased significantly from 0.6 to $0.5(22.4 \%)$ between the baseline and follow-up periods ( $p=$ 0.017) (Table 3; Supplemental Figure S1, see the electronic supplementary material). This decrease seems to have been driven by the recent oral antipsychotic subgroup, as the differences in the other two subgroups were not statistically significant. Among patients with recent oral antipsychotic use, there was a statistically significant reduction in the proportion of patients with an all-cause inpatient admission (from $36.9 \%$ to $26.2 \%$; $p=0.007$ ) and the mean number of admissions (from 0.7 to $0.5 ; p=0.027$ ), as well as a $38.5 \%$ reduction in average length of stay (from 3.7 to 2.3 days; $p=0.004)$. The proportion of patients with a mental health-related inpatient admission $(p=0.037)$ and the number of admissions per patient $(p=0.011)$ were also significantly lower in the 6-month period after compared with before AL initiation, again likely driven by changes observed in patients with recent oral antipsychotic use (Table 3). This patient subgroup also had statistically significant reductions in proportion of patients with a mental health-related inpatient admission $(p=0.002)$, mean number of mental health-related admissions per patient $(p=0.046)$, and average length of mental health-related stay $(p=0.010)$.

\subsubsection{Outpatient Utilization}

Although there were no statistically significant differences in the utilization of all-cause outpatient services before and after AL initiation overall (Supplemental Figure S1, see the electronic supplementary material), the recent oral antipsychotics subgroup experienced a significant decrease in the proportion of patients with at least one all-cause ER visit after AL initiation (from 53.8 to $44.6 \% ; p=0.029$ ). The proportion of patients with at least one mental health-related ER visit decreased significantly in the overall population, from 40.0 to $34.6 \%$ ( $p=0.025$, Table 3$)$. This decrease was likely driven by the subgroup with recent oral antipsychotic use, as this subgroup had a statistically significant reduction in this measure from 47.7 to $37.4 \%(p=0.014)$.

\subsubsection{Outpatient Prescription Drug Utilization}

The mean number of all-cause prescription fills increased significantly from 32.3 to 36.0 between baseline and followup $(p<0.001)$.

\subsection{Healthcare Costs}

Supplemental Table S1 summarizes patterns of all-cause and mental health-related healthcare costs in the 6-month period before versus after initiation with AL (see the electronic supplementary material). Figure 2 displays the net change in costs from baseline to follow-up, overall and by subgroup. Below, we summarize trends for all-cause inpatient, outpatient, and prescription drug costs. Mental health-related healthcare costs are described only if markedly different from all-cause costs.

\subsubsection{Inpatient Costs}

Across the full cohort, mean all-cause inpatient costs decreased from US $\$ 6121$ to US $\$ 3284$ ( $p=0.036$; Fig. 2 ) and mean mental health-related inpatient costs decreased from US\$4333 to US\$2803 ( $p=0.005)$ in the 6-month period after AL initiation compared with the 6 months before. All three prior antipsychotic subgroups had lower mean all-cause and mental health-related inpatient costs after AL initiation. The cohort with recent oral antipsychotic use showed the greatest cost savings and was the only subgroup with a statistically significant reduction in mental health-related inpatient costs (US\$5831 to US\$3322, $p=$ 0.007; Supplemental Table S1 and Figure S1.

\subsubsection{Outpatient Services Costs}

Mean all-cause outpatient services costs did not change significantly between the baseline and follow-up periods overall or within any prior treatment subgroup (Fig. 2). However, mental health-related outpatient services costs increased from US\$5739 to US\$6358 for the full cohort over the 6-month follow-up period ( $p=0.008$; Supplemental Table S1, see the electronic supplementary material). Among patients with recent oral antipsychotic use, all-cause ER visit costs decreased significantly from baseline to follow-up, from US $\$ 508$ to US $\$ 358$ ( $p=0.022)$.

\subsubsection{Outpatient Prescription Drug Costs}

Mean all-cause outpatient prescription costs increased from US\$5443 to US\$9565 for the full cohort between the baseline and follow-up periods $(p<0.001)$. Increases in outpatient prescription costs were observed in all subgroups (all $p<0.001$ ) and were highest among patients with no recent antipsychotic use and lowest among patients with recent LAI antipsychotic use. 


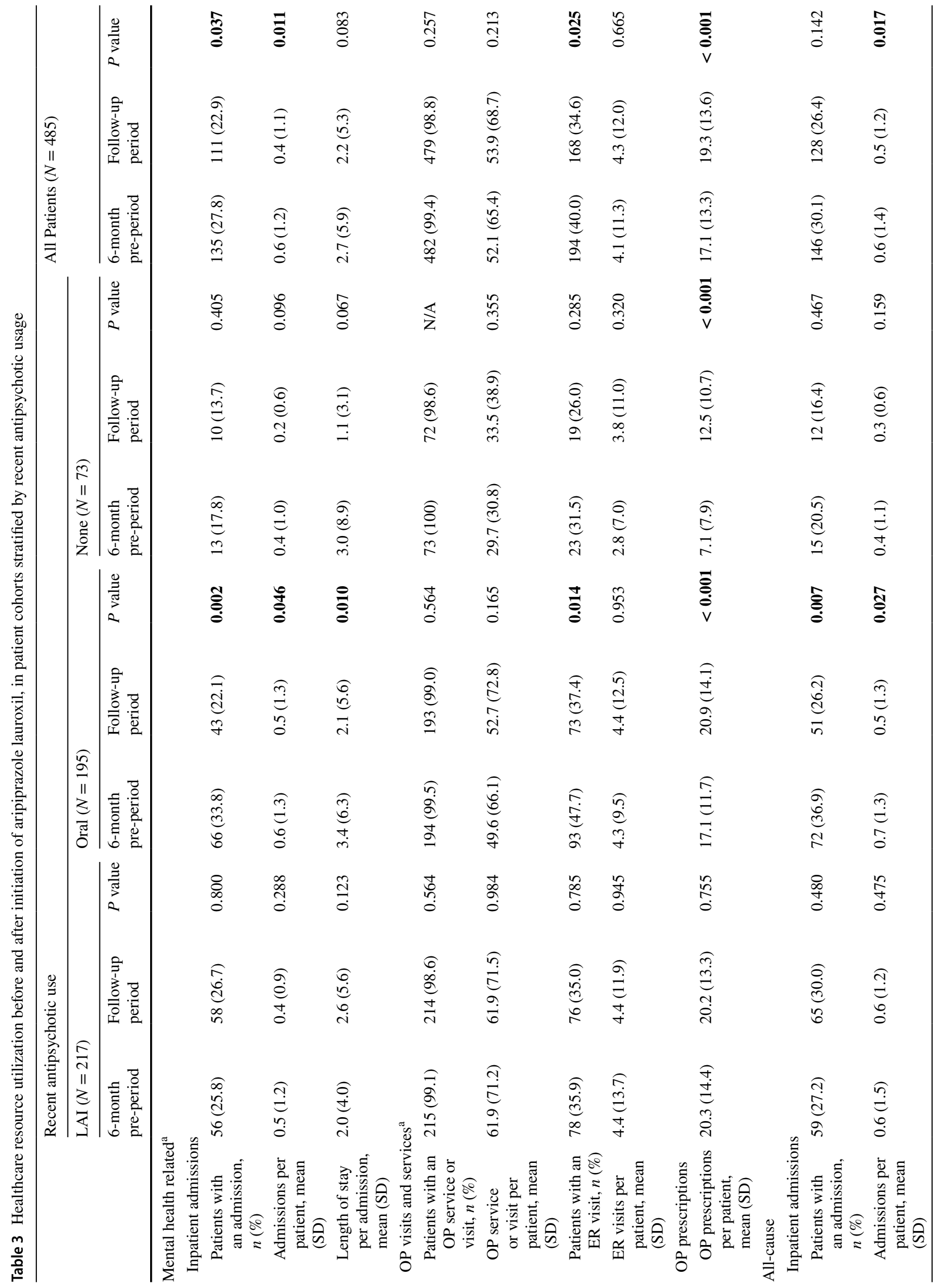




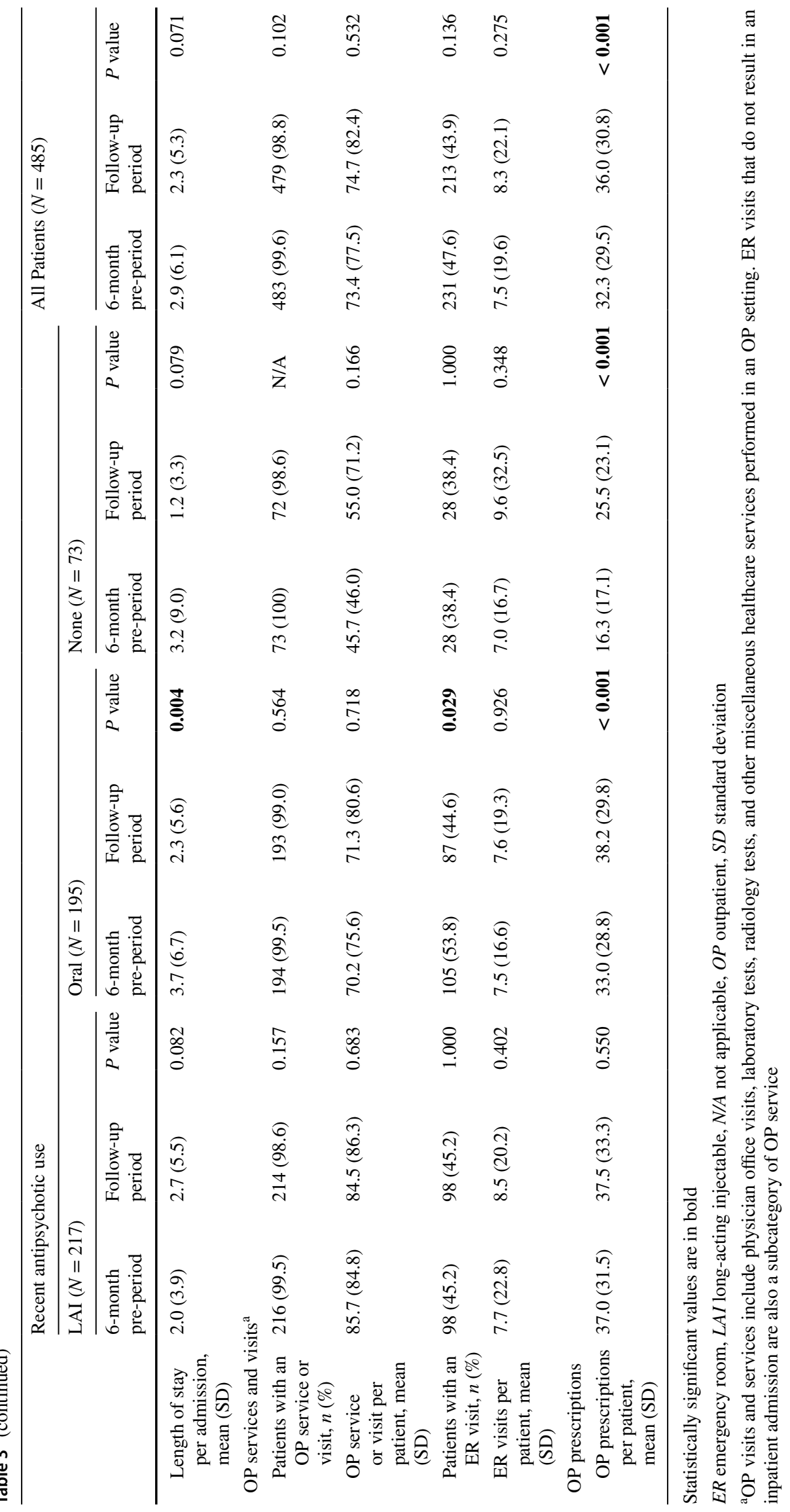


Fig. 2 Change in all-cause healthcare costs from baseline to follow-up. Mean change in cost is represented. Statistically significant findings $(p<0.05)$ are represented with an asterisk. $A P$ antipsychotic, LAI longacting injectable antipsychotic, $O P$ outpatient, USD US dollars

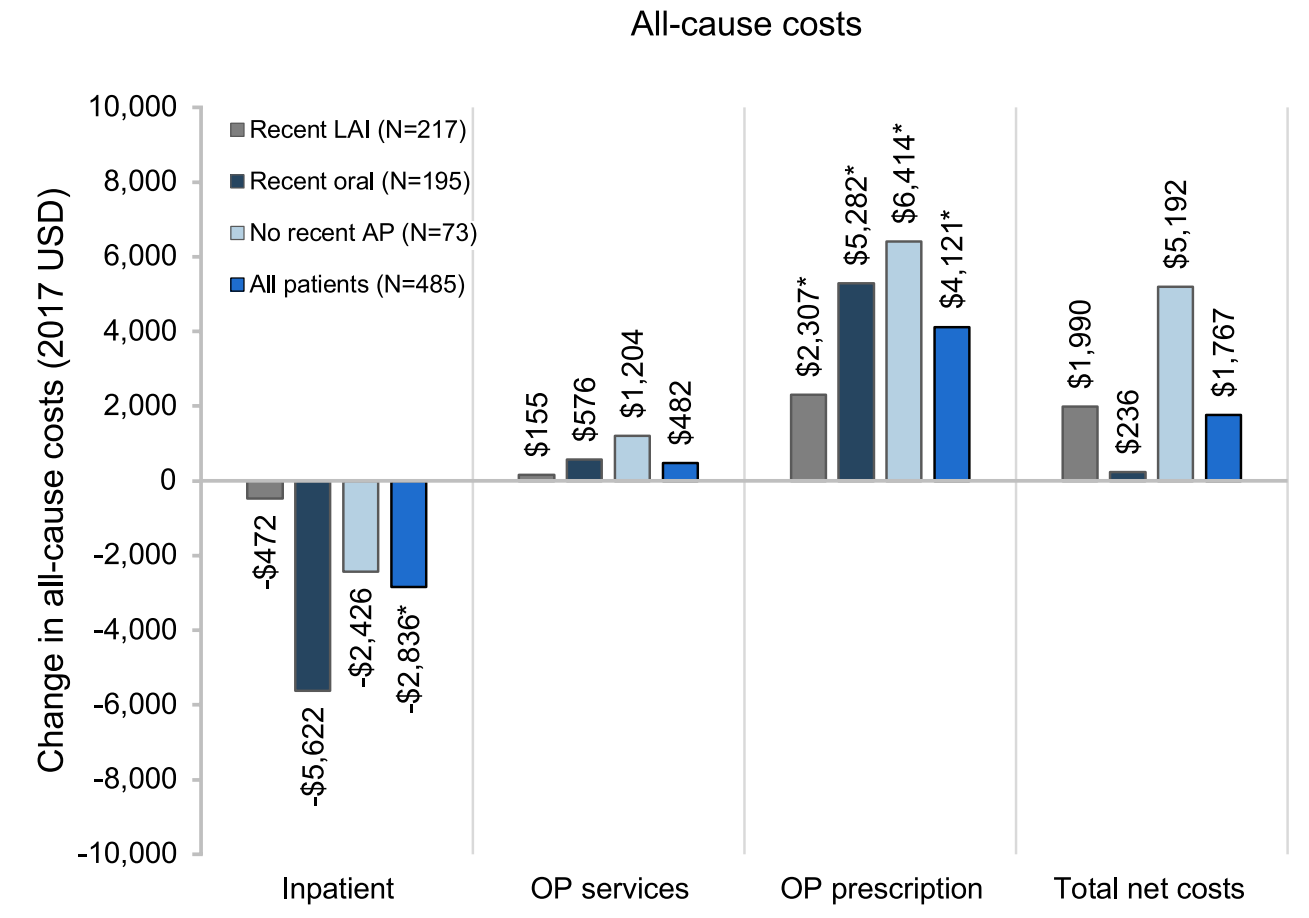

\subsubsection{Total Healthcare Costs}

When examined overall, there was no significant change in all-cause total costs between the baseline and follow-up periods (Supplemental Table S1, see the electronic supplementary material). Mental health-related total costs increased from US $\$ 14,695$ to US $\$ 17,720$ from baseline to follow-up $(p<0.001)$, driven by increases in outpatient services and prescription drugs. Increases in these categories were driven by the recent oral antipsychotic subgroup and the no recent antipsychotic use subgroup. No subgroup demonstrated a statistically significant difference in all-cause total costs. In sum, costs were observed to shift from inpatient services in the baseline period to outpatient prescriptions in the followup period with no change in total costs.

\section{Discussion}

This real-world, claims-based study compared treatment patterns, healthcare utilization, and costs among Medicaidinsured patients with schizophrenia 6 months before and 6 months after initiating AL. Over the 6-month follow-up period, patients received on average 4.6 injections of AL and were persistent for 133.4 days, which translates to drug exposure over $77 \%$ of the follow-up period. Results indicated that patients initiating AL experience significant reductions in all-cause and mental health-related inpatient admissions and proportion of patients with mental health-related ER visits. Overall, there was no significant change in total healthcare costs between the baseline and follow-up periods. Though pharmacy costs increased after initiating AL, this was offset by a reduction in inpatient costs. While the initiation of AL was neutral in terms of overall healthcare costs and resource utilization, the reduction in inpatient admissions suggests that the change to AL may have had additional benefits outside the scope of this study, considering that patients with schizophrenia who are stable on LAI antipsychotic medication may also experience improvements in quality of life and social, occupational, and psychological functioning [26] and may have a reduced risk of relapse [27].

The stability in total healthcare costs in the overall study sample was also observed in the individual subgroups defined by type of antipsychotic received in the 60 days prior to AL initiation (recent LAI antipsychotic, recent oral antipsychotic, or no recent antipsychotic). All three subgroups had a significant increase in mental health-related outpatient prescription costs after initiating AL, while total all-cause expenditures were unchanged. For each subgroup, mental health-related inpatient costs were numerically lower in the follow-up period compared with baseline, although the change reached significance only for those patients in the recent oral antipsychotic subgroup. The patients in that subgroup had significantly reduced resource-intensive acute care (inpatient and ER), while outpatient pharmacy services were increased. Several factors may have contributed to the observed differences in reductions in inpatient costs between subgroups. Patients with recent oral antipsychotic use may have been initiating an LAI antipsychotic because they were not stable on their current medication, and for these patients, 
a reduction in undetected medication gaps can result in greater stability and decreased need for hospitalization [8]. Indeed, that subgroup experienced significantly reduced all-cause and mental health-related inpatient admissions in the follow-up period. These findings are supported by results of other real-world studies that assessed healthcare costs associated with initiating once-monthly paliperidone palmitate. It was reported that post paliperidone palmitate switch, a decrease in inpatient costs was offset by an increase in pharmacy costs, resulting in similar total costs compared to pre-switch $[24,25]$. A study examining costs 6 months before versus after a switch from an oral medication to once-monthly aripiprazole monohydrate similarly reported increased pharmacy costs and reduced hospital costs [28].

In contrast with the recent oral antipsychotic subgroup, patients using LAI antipsychotics prior to initiating AL were already benefiting from uninterrupted antipsychotic treatment during the baseline period, which may have accounted for the lack of a significant reduction in inpatient costs in the follow-up period. A similar result was reported in one of two studies that used a 6-month pre/post design to assess costs associated with a switch from paliperidone palmitate once monthly to every 3 months [29]; inpatient costs and total overall costs were significantly reduced in the second study [30]. Various factors may account for the lack of significant reduction in inpatient healthcare utilization for the no recent antipsychotic subgroup. The patients in the no recent antipsychotic subgroup may have been less severely ill, requiring fewer services before or after initiating AL, or they may have been as ill as patients in the other subgroups but not seeking the services they needed. Taken together, the subgroup analysis results suggest that greater reductions in healthcare resource utilization after initiating AL could potentially be expected in less clinically stable or more severely ill patients.

Adherence to therapy with AL in the current study was similar to or exceeded adherence reported after a switch from oral antipsychotics to once-monthly paliperidone palmitate in two published studies that used the 6-month pre/post design [24, 25]. Other studies on adherence with LAI antipsychotics for the treatment of schizophrenia have assessed longer follow-up periods. One previous analysis found that $50 \%$ of Medicaid-insured schizophrenia patients initiating an LAI antipsychotic had discontinued treatment by 6.5 months and $63.2 \%$ had discontinued by 1 year [31]. Another study compared patients initiating typical and atypical LAI antipsychotics and reported that patients initiating an atypical LAI antipsychotic had a mean (SD) PDC of $0.521(0.297)$ over 1 year [32]. In our study of AL initiators, immediate discontinuation was low-only $13.8 \%$ of the cohort received a single administration over follow-up — and estimated adherence was 0.76 (a mean of 136 days) over the 6-month follow-up period. Comparisons should be interpreted with caution, however, due to potential differences in data sources, dosing intervals, and period of follow-up. Patients with the best adherence with $\mathrm{AL}$ - based on number of doses in 6 months, PDC, and rate of discontinuationwere those in the recent LAI antipsychotic use subgroup. The higher adherence rates observed among those patients with recent LAI antipsychotic use in our study suggests that adherence and persistence may improve once patients are comfortable and committed to the injections and the decision to change LAI antipsychotic could be based on tolerability or efficacy [33-35]. Patients with recent LAI antipsychotic use also tended to maintain their existing healthcare resource utilization patterns after initiating AL. This suggests that these patients have committed and/or accepted an LAI antipsychotic as a mode of administration for their antipsychotic therapy.

Together with the previous literature, these results show a reproducible trend in healthcare utilization and cost burden moving away from inpatient care with the introduction of an LAI antipsychotic. Moreover, our findings suggest that this trend is maintained among patients with prior LAI antipsychotic exposure. These results from patients initiated on AL immediately following Food and Drug Administration (FDA) approval indicate that patients stay on the drug, and that AL-with its availability of multiple dosage strengths and administration timing - may reduce reliance on inpatient utilization for management of schizophrenia, particularly among patients transitioning from an oral antipsychotic.

\subsection{Limitations}

The primary limitation of this study was that the MarketScan ${ }^{\circledR}$ Multi-State Medicaid Database does not capture inpatient medication use, and, consequently, inpatient AL use was not included in this analysis. It is therefore possible that we underestimated the number of total AL administrations and overestimated discontinuation if patients continued to receive AL during a hospital stay. In particular, there were 67 patients with a recorded discontinuation who also experienced an inpatient hospitalization over the followup period. Depending on timing, these patients may have received an AL injection during their inpatient admission, which would not have been detected given the limitations of the study database, resulting in an underestimation of time on therapy.

Another limitation of the study was that the coding system used to identify diagnoses changed in the middle of our patient identification period. Specifically, AL was first approved for use by the FDA on October 5, 2015. Our selection criteria required that patients had an appropriate diagnosis within 12 months prior to the initial AL prescription, and therefore the identification period for a schizophrenia diagnosis spanned the period from October 1, 2014, to June 30, 2017. The US Department of Health and Human Services 
mandated that all entities covered by the HIPAA transition from using ICD-9 diagnosis codes to ICD-10 codes as of October 1, 2015. ICD-9 diagnosis codes were used for all claims of service until September 30, 2015, and after that date, ICD-10 codes were used. Therefore, we had to map ICD-9 codes to the closest ICD-10 codes available to ensure the patients were comparable between the two periods.

This study used data from Medicaid-insured patients, and the findings may not be generalizable to patients without insurance or with other types of insurance. This being said, the database was selected because more than half of patients with schizophrenia report being insured through Medicaid [36]. In addition, variables such as disease severity, over-thecounter medication use, and patient health behavior are not captured and therefore could not be measured or included in our analyses. Further, although the within-patient comparisons facilitated by the pre/post study design avoid the need to adjust for patient-related factors, the design does not take into account patients' health-related changes over time and, in particular, that patients may start a new medication for schizophrenia during an exacerbation of symptoms or a relapse, a time when resource use and costs are likely to be high [37].

At the time this study was conducted, AL was new to the market. The time frame of this study captured experience with AL within 20 months of FDA approval. As clinicians gain more experience with any treatment, one might expect changes in how the treatment is used. Also, clinicians using AL shortly after approval might have different characteristics than those who wait to prescribe a new therapy. Likewise, patient characteristics or dosing decisions may change with greater cumulative experience. Therefore, the results of this study may not apply in the future, as more experience with AL accrues. Furthermore, there are now two additional formulations of AL available: a dose of $1064 \mathrm{mg}$, introduced for use as a 2-month dose interval option, and Aristada Initio, given as a one-time injection and used in combination with oral aripiprazole to start AL treatment. Future studies should examine treatment patterns, resource use, and costs once these formulations are assimilated into practice.

A final note is that overall direct cost estimates such as this one do not acknowledge the "real-world" challenges when budgets are organized in silos. For example, this analysis found that inpatient costs decreased and pharmacy costs increased. The neutrality in total costs may be of little comfort or interest to those responsible for managing (outpatient) pharmacy budgets. While not a limitation of the methods per se, the fragmentation of US payers and cost-shifting challenges are well known and may limit the usefulness of this information to payers organized in silos. On the other hand, in the absence of a fragmented system, the use of LAI antipsychotics has the potential to improve outcomes for patients (i.e., through fewer hospitalizations) without added costs.

\section{Conclusions}

This cohort of patients who initiated AL shortly after its approval were able to remain on it over the majority of the 6-month time window assessed in this analysis, with patients receiving $77 \%$ of the theoretical maximum six injections for a monthly LAI antipsychotic schedule. Patients experienced a reduction in inpatient utilization and costs, mental health-related ER utilization, and an increase in pharmacy costs in the 6-month period after AL initiation compared to the 6-month period before initiation. These differences offset each other, resulting in no significant change in total healthcare costs. Patients who used oral antipsychotics before starting AL (versus another LAI antipsychotic) had the greatest reduction in inpatient use and costs and therefore may represent opportunity for improved clinical benefits (reductions in hospitalizations) without additional costs. Taken together, the adherence rate and reduction in inpatient admissions and costs may indicate greater clinical stability for patients. Further epidemiologic and economic studies with longer follow-up periods are required to explore how more experience with AL may impact its use or the types of patients who are selected for therapy.

Supplementary Information The online version contains supplementary material available at https://doi.org/10.1007/s40263-021-00849-2.

Acknowledgements The authors received medical writing support from Jessamine Winer-Jones of IBM Watson Health, Cambridge, MA, USA, funded by Alkermes, Inc.

\section{Declarations}

Funding This study was funded by Alkermes, Inc. The open access fee was paid by Alkermes, Inc.

Conflict of interest AKO, CDG, AS, and PJW were employed by Alkermes, Inc., at the time this research was conducted. LB, KJP, and EH were employed by IBM Watson Health at the time this research was conducted and received funding from Alkermes, Inc., to conduct this study. JL is a paid consultant of Alkermes, Inc., although he was not paid for his time on this article.

Ethics approval All database records are statistically de-identified and certified to be fully compliant with US patient confidentiality requirements set forth in the Health Insurance Portability and Accountability Act of 1996. Because this study used only de-identified patient records and did not involve the collection, use, or transmittal of individually identifiable data, institutional review board approval to conduct this study was not necessary.

Consent to participate Not applicable.

Consent for publication Not applicable.

Availability of data and material The data that support the findings of this study are available from IBM Watson Health. Restrictions apply to the availability of these data, which were used under license for this study. 
Code availability Not applicable.

Author contribution JL, CG, LJB, AS, AOS, EH, KJP, PW contributed to the design of the study and interpretation of the data. AOS, EH, KJP, $\mathrm{PW}$, contributed to the data collection and data analysis. All authors critically revised the draft manuscript and approved the final content and are accountable for the work presented.

Open Access This article is licensed under a Creative Commons Attribution-NonCommercial 4.0 International License, which permits any non-commercial use, sharing, adaptation, distribution and reproduction in any medium or format, as long as you give appropriate credit to the original author(s) and the source, provide a link to the Creative Commons licence, and indicate if changes were made. The images or other third party material in this article are included in the article's Creative Commons licence, unless indicated otherwise in a credit line to the material. If material is not included in the article's Creative Commons licence and your intended use is not permitted by statutory regulation or exceeds the permitted use, you will need to obtain permission directly from the copyright holder. To view a copy of this licence, visit http://creativecommons.org/licenses/by-nc/4.0/.

\section{References}

1. GBD 2017 Disease and Injury Incidence and Prevalence Collaborators. Global, regional, and national incidence, prevalence, and years lived with disability for 354 diseases and injuries for 195 countries and territories, 1990-2017: a systematic analysis for the Global Burden of Disease Study 2017. Lancet. 2018;392(10159):1789-858.

2. Charlson FJ, Ferrari AJ, Santomauro DF, Diminic S, Stockings E, Scott JG, et al. Global epidemiology and burden of schizophrenia: findings from the Global Burden of Disease study 2016. Schizophr Bull. 2018;44(6):1195-203.

3. He H, Liu Q, Li N, Guo L, Gao F, Bai L, et al. Trends in the incidence and DALYs of schizophrenia at the global, regional and national levels: results from the Global Burden of Disease Study 2017. Epidemiol Psychiatr Sci. 2020;29:e91.

4. Chong HY, Teoh SL, Wu DB, Kotirum S, Chiou CF, Chaiyakunapruk N. Global economic burden of schizophrenia: a systematic review. Neuropsychiatr Dis Treat. 2016;12:357-73.

5. Murray CJ, Atkinson C, Bhalla K, Birbeck G, Burstein R, Chou D, et al. The state of US health, 1990-2010: burden of diseases, injuries, and risk factors. JAMA. 2013;310(6):591-608.

6. Cloutier M, Aigbogun MS, Guerin A, Nitulescu R, Ramanakumar AV, Kamat SA, et al. The economic burden of schizophrenia in the United States in 2013. J Clin Psychiatry. 2016;77(6):764-71.

7. Emsley R, Chiliza B, Asmal L, Harvey BH. The nature of relapse in schizophrenia. BMC Psychiatry. 2013;13:50.

8. Weiden PJ, Kozma C, Grogg A, Locklear J. Partial compliance and risk of rehospitalization among California Medicaid patients with schizophrenia. Psychiatr Serv. 2004;55(8):886-91.

9. Buchanan RW, Kreyenbuhl J, Kelly DL, Noel JM, Boggs DL, Fischer BA, et al. The 2009 schizophrenia PORT psychopharmacological treatment recommendations and summary statements. Schizophr Bull. 2010;36(1):71-93.

10. Biagi E, Capuzzi E, Colmegna F, Mascarini A, Brambilla G, Ornaghi A, et al. Long-acting injectable antipsychotics in schizophrenia: literature review and practical perspective, with a focus on aripiprazole once-monthly. Adv Ther. 2017;34(5):1036-48.

11. Valenstein M, Ganoczy D, McCarthy JF, Myra Kim H, Lee TA, Blow FC. Antipsychotic adherence over time among patients receiving treatment for schizophrenia: a retrospective review. J Clin Psychiatry. 2006;67(10):1542-50.

12. Bright CE. Measuring medication adherence in patients with schizophrenia: an integrative review. Arch Psychiatr Nurs. 2017;31(1):99-110.

13. Correll CU, Citrome L, Haddad PM, Lauriello J, Olfson M, Calloway SM, et al. The use of long-acting injectable antipsychotics in schizophrenia: evaluating the evidence. J Clin Psychiatry. 2016;77(Suppl 3):1-24.

14. Cañas F, Alptekin K, Azorin JM, Dubois V, Emsley R, García $\mathrm{AG}$, et al. Improving treatment adherence in your patients with schizophrenia: the STAY initiative. Clin Drug Investig. 2013;33(2):97-107.

15. Lally $\mathbf{J}$, MacCabe JH. Antipsychotic medication in schizophrenia: a review. Br Med Bull. 2015;114(1):169-79.

16. Velligan DI, Weiden PJ, Sajatovic M, Scott J, Carpenter D, Ross $\mathrm{R}$, et al. The expert consensus guideline series: adherence problems in patients with serious and persistent mental illness. J Clin Psychiatry. 2009;70(Suppl 4):1-46 ((quiz 7-8)).

17. Velligan DI, Sajatovic M, Hatch A, Kramata P, Docherty JP. Why do psychiatric patients stop antipsychotic medication? A systematic review of reasons for nonadherence to medication in patients with serious mental illness. Patient Prefer Adherence. 2017;11:449-68.

18. Llorca PM, Bobes J, Fleischhacker WW, Heres S, Moore N, BentEnnakhil N, et al. Baseline results from the European non-interventional Antipsychotic Long acTing injection in schizOphrenia (ALTO) study. Eur Psychiatry. 2018;52:85-94.

19. Weiden PJ, Turkington D. Assessment and management of medication nonadherence in schizophrenia. In: Lieberman JA, Murray RM, editors. Comprehensive care of schizophrenia: a textbook of clinical management. 2nd ed. New York: Oxford University Press; 2012. p. 219-43.

20. Weiden PJ, Solari H, Kim S, Bishop JR. Long-acting injectable antipsychotics and the management of nonadherence. Psychiatr Ann. 2011;41(5):271278.

21. Aristada [package insert]. Waltham, MA: Alkermes, Inc.; 2020 February 2020.

22. Deyo RA, Cherkin DC, Ciol MA. Adapting a clinical comorbidity index for use with ICD-9-CM administrative databases. J Clin Epidemiol. 1992;45(6):613-9.

23. US Bureau of Labor Statistics. Consumer Price Index Detailed Report Tables: United States Department of Labor; 2020. https:// www.bls.gov/cpi/home.htm.

24. Patel C, Emond B, Lafeuille MH, Côté-Sergent A, Lefebvre P, Tandon N, et al. Real-world analysis of switching patients with schizophrenia from oral risperidone or oral paliperidone to oncemonthly paliperidone palmitate. Drugs Real World Outcomes. 2020;7(1):19-29.

25. Patel C, Khoury AE, Huang A, Wang L, Bashyal R. Healthcare resource utilization and costs among patients with schizophrenia switching from oral risperidone/paliperidone to once-monthly paliperidone palmitate: a Veterans Health Administration claims analysis. Curr Ther Res Clin Exp. 2020;92:100587.

26. Lloyd K, Latif MA, Simpson S, Shrestha KL. Switching stable patients with schizophrenia from depot and oral antipsychotics to long-acting injectable risperidone: efficacy, quality of life and functional outcome. Hum Psychopharmacol. 2010;25(3):243-52.

27. Gaebel W, Schreiner A, Bergmans P, de AR, Rouillon F, Cordes J, et al. Relapse prevention in schizophrenia and schizoaffective disorder with risperidone long-acting injectable vs quetiapine: results of a long-term, open-label, randomized clinical trial. Neuropsychopharmacology. 2010;35(12):2367-77.

28. Wilson M, Gutierrez B, Offord SJ, Blanchette CM, Eramo A, Earnshaw $\mathrm{S}$, et al. Inpatient resource use and costs associated with 
switching from oral antipsychotics to aripiprazole once-monthly for the treatment of schizophrenia. Drugs Context. 2016;5:212273.

29. Emond B, El Khoury AC, Patel C, Pilon D, Morrison L, Zhdanava $\mathrm{M}$, et al. Real-world outcomes post-transition to onceevery-3-months paliperidone palmitate in patients with schizophrenia within US commercial plans. Curr Med Res Opin. 2019;35(3):407-16.

30. DerSarkissian M, Lefebvre P, Joshi K, Brown B, Lafeuille MH, Bhak RH, et al. Health care resource utilization and costs associated with transitioning to 3-month paliperidone palmitate among US veterans. Clin Ther. 2018;40(9):1496-508.

31. Greene M, Yan T, Chang E, Hartry A, Touya M, Broder MS. Medication adherence and discontinuation of long-acting injectable versus oral antipsychotics in patients with schizophrenia or bipolar disorder. J Med Econ. 2018;21(2):127-34.

32. Pilon D, Joshi K, Tandon N, Lafeuille MH, Kamstra RL, Emond $\mathrm{B}$, et al. Treatment patterns in Medicaid patients with schizophrenia initiated on a first- or second-generation long-acting injectable versus oral antipsychotic. Patient Prefer Adherence. 2017;11:619-29.
33. Baser O, Xie L, Pesa J, Durkin M. Healthcare utilization and costs of Veterans Health Administration patients with schizophrenia treated with paliperidone palmitate long-acting injection or oral atypical antipsychotics. J Med Econ. 2015;18(5):357-65.

34. Shah A, Xie L, Kariburyo F, Zhang Q, Gore M. Treatment patterns, healthcare resource utilization and costs among schizophrenia patients treated with long-acting injectable versus oral antipsychotics. Adv Ther. 2018;35(11):1994-2014.

35. Peng X, Ascher-Svanum H, Faries D, Conley RR, Schuh KJ. Decline in hospitalization risk and health care cost after initiation of depot antipsychotics in the treatment of schizophrenia. ClinicoEcon Outcomes Res. 2011;3:9-14.

36. Khaykin E, Eaton WW, Ford DE, Anthony CB, Daumit GL. Health insurance coverage among persons with schizophrenia in the United States. Psychiatr Serv. 2010;61(8):830-4.

37. Faries DE, Nyhuis AW, Ascher-Svanum H. Methodological issues in assessing changes in costs pre- and post-medication switch: a schizophrenia study example. Cost Eff Resour Alloc. 2009;7:11. 